\title{
Impuestos selectivos al consumo de bebidas azucaradas en América Latina y el Caribe*
}

\author{
Rosa Carolina Sandoval, ${ }^{1}$ Maxime Roche, ${ }^{1}$ Itziar Belausteguigoitia, ${ }^{2}$ Miriam Alvarado, ${ }^{3}$ Luis Galicia, ${ }^{4}$ \\ Fabio S. Gomes ${ }^{1}$ y Guillermo Paraje ${ }^{5}$
}

Forma de citar

Sandoval RC, Roche M, Belausteguigoitia I, Alvarado M, Galicia L, Gomes FS, et al. Impuestos selectivos al consumo de bebidas azucaradas en América Latina y el Caribe. Rev Panam Salud Publica. 2021;45:e124 https://doi.org/10.26633/ RPSP.2021.124

RESUMEN

Objetivo. Caracterizar el diseño de los impuestos selectivos al consumo de bebidas azucaradas en América Latina y el Caribe, y evaluar las oportunidades de aumentar su impacto en el consumo y la salud.

Métodos. Se llevó a cabo una búsqueda y una evaluación exhaustivas de legislaciones vigentes a marzo del 2019, recopiladas mediante las herramientas de seguimiento ya existentes de la Organización Panamericana de la Salud y de la Organización Mundial de la Salud, fuentes secundarias, así como mediante una encuesta a ministerios de finanzas. El análisis se centró en el tipo de productos gravados y la estructura y la base de estos impuestos selectivos.

Resultados. De los 33 países evaluados, en 21 se aplican impuestos selectivos al consumo de bebidas azucaradas. En siete países también se aplican impuestos selectivos sobre el agua embotellada y en al menos cuatro, se aplican tales impuestos sobre las bebidas lácteas azucaradas. Diez de estos impuestos selectivos al consumo son de tipo ad valorem con algunas bases imponibles fijadas en las primeras etapas de la cadena de valor, siete son de tipo específico y cuatro son de estructura combinada o mixta. En tres países se aplican impuestos selectivos al consumo en función de la concentración de azúcares del producto.

Conclusiones. Si bien el número de países en que se aplican impuestos selectivos al consumo de bebidas azucaradas es prometedor, existe una gran heterogeneidad en su diseño en cuanto a la estructura, la base imponible y los productos gravados. Se podrían aprovechar aún más los impuestos selectivos existentes para tener un mayor impacto sobre el consumo de bebidas azucaradas y la salud si se incluyeran todas las categorías de bebidas azucaradas, excluyendo el agua embotellada, y si se recurriera más a impuestos específicos ajustados regularmente según la inflación y basados posiblemente en la concentración de azúcares del producto. Todos los países se beneficiarían si hubiera mayor orientación. Futuras investigaciones deberían tener como objetivo abordar esta brecha.

Palabras clave Enfermedades no transmisibles; política nutricional; economía de la salud; obesidad; legislación como asunto.

2 Universidad de Lancaster, Lancaster, Reino Unido.

3 Universidad de Cambridge, Cambridge, Reino Unido.

4 Ministerio de Salud Pública del Uruguay, Montevideo, Uruguay.

5 Universidad Adolfo Ibáñez, Santiago, Chile.

1 Organización Panamericana de la Salud, Washington, D.C. Estados Unidos de

América. $\square$ Maxime Roche, maxime.roche@edhec.com 
En toda América Latina y el Caribe, como en casi todo el mundo, la carga de enfermedades no transmisibles (ENT) es elevada y sigue en aumento. Al año 2016, tres de cada cuatro muertes en esta región fueron provocadas por ENT, y de ellas, $43 \%$ se registraron en personas de menos de 70 años (1). Las cuatro ENT principales (cáncer, diabetes mellitus, enfermedades cardiovasculares y enfermedades respiratorias crónicas) representaron 2,15 millones de muertes, es decir, tres cuartas partes de todas las muertes provocadas por ENT (1). Se ha estimado que, a escala mundial, el costo de esas cuatro ENT ascendió a US\$ 3,8 billones en el 2010, y las proyecciones lo sitúan en US\$ 7 billones para el 2030 (2).

La Organización Mundial de la Salud (OMS) ha establecido una serie de soluciones costo-eficaces y basadas en la evidencia para prevenir y controlar las ENT $(3,4)$. Entre ellas cabe mencionar la recomendación de reducir el consumo de azúcares mediante la tributación eficaz de las bebidas azucaradas (3-5). Estas se definen como todos los tipos de bebidas sin alcohol que contienen azúcares libres, entre las que se incluyen los refrescos gaseosos o no gaseosos, los jugos y bebidas de frutas y verduras, los concentrados líquidos y en polvo, el agua saborizada, las bebidas energizantes y deportivas, el té listo para beber, el café listo para beber y las bebidas lácteas saborizadas. Estas bebidas proporcionan un valor nutritivo limitado, pueden conducir a una ingesta calórica excesiva y se las ha relacionado con resultados de salud negativos (6). Es más, se las ha señalado como el principal factor de la epidemia de obesidad (7) y en exámenes sistemáticos se ha demostrado que su consumo se vincula con el aumento de peso en niños y adultos (8), un riesgo mayor de hipertensión y cardiopatías coronarias (9), y una mayor incidencia de diabetes de tipo 2 (10).

América Latina y el Caribe tiene la mayor mortalidad absoluta del mundo relacionada con el consumo de bebidas azucaradas, con unas 159 muertes por millón de adultos (en comparación con 48 muertes por millón de adultos a nivel mundial), de las que $80 \%$ se vinculan con la diabetes (11). El consumo diario medio de bebidas azucaradas por adulto en América Latina y el Caribe es el más alto del mundo, en particular en el Caribe y Centroamérica (en promedio 1,93 y 1,61 raciones diarias de ocho onzas por adulto, respectivamente, en comparación con 0,58 a nivel mundial) (12). Además, los niveles de obesidad son mayores que en cualquier otra parte del mundo (13). La aplicación de impuestos selectivos sobre las bebidas azucaradas es una opción de política prometedora para reducir el consumo de dichas bebidas y la carga de las ENT. Varias evaluaciones de los impuestos selectivos sobre las bebidas azucaradas aplicados en América Latina y el Caribe han demostrado que se vinculan con reducciones en las ventas y el consumo de dichas bebidas (14-17). Por ejemplo, se ha demostrado que el impuesto selectivo de 1 peso por litro introducido en México en el 2014 redujo las compras de bebidas azucaradas en un promedio anual de 7,6\% durante los dos primeros años posteriores a su establecimiento (14). El impuesto selectivo ad valorem en dos niveles adoptado en Chile se ha vinculado con una reducción de 3,4\% en el volumen mensual promedio adquirido por hogar en cuanto a las bebidas azucaradas con la mayor concentración de azúcares (15). El impuesto selectivo ad valorem en Barbados se ha vinculado con una reducción de $4,3 \%$ en las ventas de bebidas azucaradas durante su primer año (16). Por último, en un estudio de simulación se constató que tan solo en México se prevé que el actual impuesto selectivo sobre las bebidas azucaradas prevendrá entre 86.000 y 134.000 casos de diabetes de tipo 2 para el 2030 y reducirá la prevalencia de la obesidad en $2,5 \%$, lo cual destaca el potencial que tienen los impuestos selectivos sobre las bebidas azucaradas de contribuir a los esfuerzos para prevenir las ENT (17).

Centramos nuestro análisis particularmente en los impuestos selectivos, pues tienen el mayor potencial, desde la óptica de la salud, en comparación con otros tipos de gravámenes indirectos (por ejemplo, impuestos sobre las ventas aplicados en el punto de compra [el cajero], o sobre el valor agregado). En efecto, los impuestos selectivos permiten a los responsables de las políticas enfocarse en determinados productos y aumentar su precio, con lo cual los vuelven relativamente menos asequibles que otros bienes y servicios (18). Un impuesto selectivo se aplica sobre un bien determinado, importado o de producción local, y puede ser de monto específico (basado en el volumen de la bebida o su contenido de azúcar; por ejemplo, US\$ 0,10 por litro) o ad valorem (basado en un porcentaje del valor de la bebida; por ejemplo, 10\% del precio de fábrica) (19). Se ha dicho que los impuestos selectivos sobre las bebidas azucaradas ofrecen un beneficio triple para los gobiernos, porque 1) mejoran la salud de la población, 2) generan ingresos tributarios y 3) pueden reducir los correspondientes costos de atención de salud y pérdidas de productividad a largo plazo $(20,21)$.

Si bien es posible que haya enseñanzas de la tributación del tabaco que también puedan aplicarse en el caso de las bebidas azucaradas, la orientación en cuanto a las mejores prácticas relativas a la tributación de dichas bebidas todavía se está elaborando. La mayoría de las evaluaciones de los impuestos selectivos sobre las bebidas azucaradas se centran únicamente en un país a la vez y se concentran en los impuestos recientemente implementados, aplicados con fundamentos de salud explícitos. Al momento de realizarse el presente análisis no estaba claro cuántos países aplican impuestos selectivos sobre las bebidas azucaradas en toda América Latina y el Caribe, o de qué manera estos varían en su diseño. Es importante caracterizar de manera integral el entorno de los actuales impuestos selectivos sobre las bebidas azucaradas a nivel de país, debido a lo siguiente: 1) el alcance de la epidemia mundial de las ENT, 2) el reconocimiento de los impuestos selectivos sobre las bebidas azucaradas como intervención costo-eficaz y basada en la evidencia, y 3) el mayor interés de los responsables de las políticas en distintos lugares del mundo en modificar o introducir dichos impuestos.

Los objetivos de este análisis son ofrecer la primera revisión exhaustiva de alcance regional de los impuestos selectivos sobre las bebidas azucaradas en América Latina y el Caribe, caracterizar la actual aplicación de dichos impuestos y evaluar las oportunidades de mejorar su impacto sobre el consumo de bebidas azucaradas y la salud.

\section{MATERIALES Y MÉTODOS}

Efectuamos una búsqueda integral de la legislación (decretos, leyes, leyes sobre impuestos selectivos y edictos y decretos-ley) sobre los impuestos selectivos aplicados a las bebidas no alcohólicas en los 33 Estados Miembros de América Latina y el Caribe de la Organización Panamericana de la Salud (OPS), Oficina Regional de la OMS para la Región de las Américas. Examinamos la legislación ya recopilada a través de las herramientas de seguimiento existentes de la OPS/OMS — el examen de las políticas de nutrición a escala mundial de la OMS, el 
informe de la OMS sobre la epidemia mundial de tabaquismo, el Sistema Mundial de Información sobre el Alcohol y la Salud de la OMS y la encuesta de capacidad de país de las ENT de la OPS - y realizamos búsquedas en los sitios web de distintos parlamentos y ministerios de economía y también en bases de datos jurídicas. Por último, mediante una encuesta realizada entre marzo y diciembre del 2019 (en adelante, encuesta de la OPS sobre los impuestos a las bebidas azucaradas) se solicitó información sobre los impuestos aplicados a las bebidas no alcohólicas directamente a los profesionales designados oficialmente de los ministerios de economía. La encuesta fue completada por 27 Estados Miembros de la OPS de América Latina y el Caribe (todos excepto Argentina, Bahamas, el Estado Plurinacional de Bolivia, Costa Rica, Haití y Nicaragua).

\section{Criterios de inclusión}

Buscamos impuestos selectivos aplicados a las bebidas sin alcohol para identificar y analizar los aplicados a las bebidas azucaradas, ya fuera que excluyeran o no a las bebidas no azucaradas como el agua embotellada y las bebidas endulzadas artificialmente. Incluimos legislación en la que se indica la estructura de tasas de los impuestos selectivos, así como leyes en las que se describe la base imponible sobre la cual se aplican las tasas de dichos impuestos. Los datos presentados se basan en legislación vigente al 31 de marzo del 2019.

\section{Extracción de datos}

Extrajimos la siguiente información de la legislación de cada país: nombre, año de la actualización más reciente, estructura tributaria (ad valorem, monto específico, mixta o combinada) y base imponible. En el caso de los impuestos ad valorem, evaluamos si la base imponible se define en los primeros tramos de la cadena de valor (por ejemplo, precio de fábrica) o en tramos subsiguientes (por ejemplo, precio minorista). En relación con los impuestos de monto específico, evaluamos si se ajustan automáticamente en función de la inflación o de otros indicadores económicos.

Además, elaboramos un grupo sencillo de indicadores para reflejar la heterogeneidad de diseño de los impuestos selectivos en los distintos países. Evaluamos si el diseño es uniforme o escalonado, si el contenido de azúcar se usa como base imponible, y si el impuesto se aplica sobre el agua embotellada, para captar cualquier diferenciación entre las bebidas azucaradas y las no azucaradas. Por último, evaluamos si la definición de productos imponibles incluye las bebidas lácteas azucaradas, las energizantes y los polvos, concentrados o jarabes usados para hacer bebidas azucaradas mediante el agregado de agua con o sin gas, a fin de evaluar si cada impuesto se aplica a una amplia gama de bebidas azucaradas o si incluye lagunas fiscales que puedan promover sustituciones no deseadas y elusión fiscal.

\section{RESULTADOS}

Constatamos que al mes de marzo del 2019, 21 Estados Miembros de la OPS de América Latina y el Caribe aplicaban impuestos selectivos sobre las bebidas azucaradas, con una proporción menor en el Caribe (6/13, información no disponible en el caso de Haití) que en América Latina (15/19). Once países (Colombia, Cuba, la República Dominicana, la República Bolivariana de
Venezuela y la mayoría de los países del Caribe) no aplicaban un impuesto selectivo sobre dichas bebidas (cuadro 1).

\section{Productos imponibles}

Los países que aplican impuestos selectivos sobre las bebidas azucaradas definen los productos imponibles de diferentes maneras. Todos los países del Caribe y algunos de los latinoamericanos (en su mayoría en Centroamérica) usan códigos arancelarios armonizados —nomenclatura internacional estandarizada para clasificar los productos objeto de comercio- para definir los productos imponibles, si bien el rango de códigos arancelarios incluidos es amplio. Es importante destacar que si bien algunos de esos códigos se definen en función de los azúcares añadidos (por ejemplo, 22.02: “Agua, incluidas el agua mineral y la gaseada, con adición de azúcar u otro edulcorante"), otros no (23), y es posible que los países deban elaborar códigos específicos más detallados para diferenciar entre bebidas azucaradas. La mayoría de los demás países latinoamericanos han elaborado definiciones de los productos imponibles sobre la base del tipo de bebida.

De todos los países que aplican impuestos selectivos sobre las bebidas azucaradas, siete los aplican también al agua embotellada. Al menos 14 incluyen uno o varios de los siguientes productos en su lista de productos imponibles: polvos, concentrados o jarabes usados para hacer bebidas azucaradas agregando agua gaseosa o no gaseosa. Todos los países que establecen impuestos selectivos sobre las bebidas azucaradas los aplican sobre las bebidas energizantes. Por último, por lo menos cuatro (Barbados, Panamá, Perú y San Vicente y las Granadinas) aplican impuestos selectivos sobre las bebidas lácteas azucaradas (cuadro 2).

\section{Estructura tributaria}

Los actuales impuestos selectivos sobre las bebidas azucaradas se basan en una variada gama de estructuras tributarias. Diez países usan exclusivamente impuestos ad valorem y siete usan impuestos de monto específico. Dominica y Ecuador utilizan una estructura de impuesto selectivo combinada. Dominica aplica un impuesto ad valorem sobre las bebidas azucaradas excepto las gaseosas, que están sujetas a un impuesto de monto específico. Ecuador aplica uno de monto específico sobre las bebidas azucaradas con una concentración de azúcar por encima de un umbral especificado y otro ad valorem sobre las bebidas azucaradas situadas por debajo de aquel umbral. Todas las bebidas energizantes (independientemente de su concentración de azúcar) están gravadas con el impuesto ad valorem. Por último, El Salvador y México usan una estructura de impuesto selectivo mixta sobre las bebidas energizantes. México utiliza principalmente uno de monto específico y aplica otro ad valorem adicional a las bebidas energizantes, mientras que El Salvador utiliza principalmente un impuesto ad valorem y aplica otro adicional de monto específico a las bebidas energizantes (cuadro 1).

En cinco países (Estado Plurinacional de Bolivia, Costa Rica, Ecuador, Honduras y México) de los once que incluyen un componente de monto específico, la legislación estipula un ajuste automático periódico del impuesto selectivo correspondiente (cuadro 2).

Once de los impuestos selectivos sobre las bebidas azucaradas establecidos en América Latina y el Caribe aplican tasas 
CUADRO 1. Resumen de los impuestos selectivos sobre las bebidas azucaradas en América Latina y el Caribe (sobre la base de la legislación vigente al 31 de marzo del 2019)

\begin{tabular}{|c|c|c|c|c|}
\hline País & $\begin{array}{l}\text { Aplica impuestos } \\
\text { selectivos sobre las } \\
\text { bebidas azucaradas }\end{array}$ & Estructura tributaria & $\begin{array}{l}\text { Año de la última } \\
\text { actualización de la } \\
\text { legislación }\end{array}$ & Legislación \\
\hline \multicolumn{5}{|l|}{ América Latina } \\
\hline Bolivia (Estado Plurinacional de) & Sí & Monto específico & 2018 & $\begin{array}{l}\text { Directorio Actualización de la Alícuotas Específicas del ICE } \\
\text { para la gestión } 2019 \text { N } 101800000031 \\
\text { Decreto Supremo } 0744 \text { de diciembre } 2010\end{array}$ \\
\hline Chile & Sí & Ad valorem & 2017 & Ley N 21.045, D.0. 3 nov 2017 Decreto de ley N 825 \\
\hline Colombia & No & & & \\
\hline Costa Rica & Sí & Monto específico & 2018 & $\begin{array}{l}\text { Ley de Simplificación y Eficiencia Tributarias N } 8114 \\
\text { Decreto Ejecutivo N } 41495 \text { de diciembre } 2018\end{array}$ \\
\hline Cuba & No & & & \\
\hline Ecuador & Sí & Combinado ${ }^{\mathrm{b}}$ & 2016 & Ley Orgánica para el Equilibrio de las Finanzas Públicas, 2016 \\
\hline Honduras & Sí & Monto específico & 2019 & Acuerdo Número 163-2019 \\
\hline México & Sí & $\begin{array}{l}\text { Monto específico } \\
\text { (mixto sobre } \\
\text { bebidas } \\
\text { energizantes) }{ }^{c}\end{array}$ & 2018 & $\begin{array}{l}\text { Ley del impuesto Especial sobre Producción y Servicios, } \\
\text { DOF 28-12-2018 }\end{array}$ \\
\hline Nicaragua & Sí & Ad valorem & 2019 & Ley de Concertación Tributaria N 987 \\
\hline Panamá & Sí & Ad valorem & 1995 & Ley N 45, 14 nov 1995 \\
\hline Paraguay & Sí & Ad valorem & 2015 & Ley N 5538/15; Ley N 125/91 \\
\hline Perú & Sí & Ad valorem & 2018 & $\begin{array}{l}\text { Decreto Supremo N 091-2018-EF } \\
\text { Título II - Decreto Supremo N 055-99-EF }\end{array}$ \\
\hline Rep. Dominicana & No & & & \\
\hline \multicolumn{5}{|l|}{ Caribe } \\
\hline Dominica & Sí & Combinado ${ }^{\mathrm{b}}$ & 2015 & $\begin{array}{l}\text { Excise Tax (Amendment), SRO N } 28 \text { of } 2015 \text { Excise } \\
\text { Tax Act 8, } 2005\end{array}$ \\
\hline Granada & No & & & \\
\hline Guyana & No & & & \\
\hline Haitía $^{2}$ & $\ldots$ & & & \\
\hline Jamaica & No & & & \\
\hline Saint Kitts y Nevis & Sí & Ad valorem & 2010 & Excise Tax Act No. 4, 2010 \\
\hline San Vicente y las Granadinas & Sí & Ad valorem & 2009 & Excise Tax Act Chapter 430 SR0 2 \\
\hline Santa Lucía & No & & & \\
\hline Suriname & Sí & Monto específico & 2006 & S.B. 2006 no. 27 wijz. Wet Accijns Alcoholvrije Dranken \\
\hline Trinidad y Tabago & No & & & \\
\hline
\end{tabular}

a.: información no disponible

"Haiti: El país no participó en la encuesta de la OPS sobre los impuestos a las bebidas azucaradas en el 2019. En nuestra búsqueda de legislación encontramos una ley de 1971 sobre impuestos selectivos (“Loi sur le Droit d’Accise, du 21 octobre 1971") que establece un impuesto selectivo de monto específico sobre las bebidas gaseosas tanto importadas como de producción local. Sin embargo, en un informe posterior de la Organización Mundial del Comercio se indica que a junio del 2015 dicho impuesto tenía una estructura diferente para esas bebidas según fueran importadas (de monto especíico) o de producción local (ad valorem), lo cual podría constituir una violación del tratamiento
nacional (22). No encontramos legislación ni información más reciente sobre ese impuesto. Ante su potencial naturaleza discriminatoria por diferenciar entre bebidas importadas o de producción local, y en vista de la falta de información, nacional (22). No encontramos legislación ni información mas reciente sobre ese impuesto. Ante su potencial naturaleza discriminatoria por diferenciar entre bebidas importadas o de producción local, y en vista de la falta de incidimos no incluirlo en nuestro análisis.
decion

' Combinado: Por lo menos un tipo de bebida no alcohólica está gravado con un impuesto selectivo ad valorem y por lo menos otro tipo lo está con uno de monto específico. Ningún tipo de bebida está gravado con ambos.

- Mixto: Por lo menos un tipo de bebida no alcohólica está gravado tanto con un impuesto selectivo ad valorem como con uno de monto específico. En El Salvador y México únicamente las bebidas energizantes están gravadas por un

sistema de impuesto selectivo mixto.

d Uruguay: El impuesto selectivo sobre las bebidas azucaradas está estructurado como impuesto ad valorem aplicado sobre una base imponible de montos fijos- "precios fictos"-por volumen, que varían por tipo de bebida, con lo cual funciona de hecho como un impuesto específico, y así se lo ha clasificado en el presente análisis.

Fuente: Preparado por los autores sobre la base de los datos del estudio. 
CUADRO 2. Información acerca del diseño de los impuestos selectivos sobre las bebidas azucaradas en América Latina y el Caribe (sobre la base de la legislación vigente al 31 de marzo del 2019)

\begin{tabular}{|c|c|c|c|c|c|c|}
\hline $\begin{array}{l}\text { País que aplica impuestos } \\
\text { selectivos sobre las } \\
\text { bebidas azucaradas }\end{array}$ & $\begin{array}{l}\text { Los aplica al agua } \\
\text { embotellada }\end{array}$ & $\begin{array}{c}\text { Los aplica a } \\
\text { los polvos, } \\
\text { concentrados o } \\
\text { jarabes }{ }^{e}\end{array}$ & $\begin{array}{l}\text { Los aplica a } \\
\text { las bebidas } \\
\text { energi-zantes }\end{array}$ & $\begin{array}{l}\text { Los aplica a las } \\
\text { bebidas lácteas } \\
\text { azucaradas } \\
\text { (código arancelario } \\
\text { armonizado: } \\
\text { 040299) }\end{array}$ & $\begin{array}{l}\text { Base imponible ad } \\
\text { valorem para bebidas } \\
\text { de producción local }\end{array}$ & $\begin{array}{ccc}\begin{array}{c}\text { Ajuste automático } \\
\text { del impuesto }\end{array} & \begin{array}{l}\text { Impuesto } \\
\text { selectivo }\end{array} & \begin{array}{c}\text { Tasa } \\
\text { tributaria }\end{array} \\
\text { selectivo de } & \text { basado en el } & \text { uniforme } \\
\text { monto especifico } & \text { contenido de } & \text { (No }= \\
\text { según la } & \text { azúcar } & \text { escalonada) } \\
\text { inflación u otros } & & \\
\text { indicadores } & & \\
\text { económicose } & & \end{array}$ \\
\hline
\end{tabular}

Impuesto selectivo con estructura ad valorem

\begin{tabular}{|c|c|c|c|c|c|c|c|c|}
\hline Argentina & Sí & Sí & Sí & $\ldots$ & $\begin{array}{l}\text { Precio minorista } \\
\text { excluido IVA }\end{array}$ & NA & No & No \\
\hline Barbados & No & Sí & Sí & Sí & Precio de fábrica & NA & No & Sí \\
\hline Brasil & $\mathrm{No}^{\mathrm{d}}$ & Sí & Sí & No & Precio de fábrica & NA & No & Sí \\
\hline Chile & No & Sí & Sí & No & $\begin{array}{l}\text { Precio minorista } \\
\text { excluido IVA }\end{array}$ & NA & Síi & No \\
\hline Nicaragua & Sí & Sí & Sí & $\ldots$ & Precio minorista & NA & No & No \\
\hline Panamá & No & Sí & Sí & Sí & Precio minorista & NA & No & Sí \\
\hline Paraguay & No & No & Sí & No & Precio de fábrica & NA & No & Sí \\
\hline Perú & No & No & Sí & Sí & $\begin{array}{l}\text { Precio minorista } \\
\text { excluido IVA e imp. } \\
\text { selectivo }\end{array}$ & NA & Síi & No \\
\hline Saint Kitts y Nevis & No & No & Sí & No & $\begin{array}{l}\text { Precio minorista } \\
\text { excluido IVA }\end{array}$ & NA & No & Sí \\
\hline $\begin{array}{l}\text { San Vicente y las } \\
\text { Granadinas }\end{array}$ & No & No & Sí & Sí & $\begin{array}{l}\text { Precio minorista } \\
\text { excluido IVA }\end{array}$ & NA & No & Sí \\
\hline \multicolumn{9}{|c|}{ Impuesto selectivo con estructura de monto específico } \\
\hline Belice & Sí & No & Sí & No & NA & No & No & Sí \\
\hline $\begin{array}{l}\text { Bolivia (Estado } \\
\text { Plurinacional de) }\end{array}$ & No & $\ldots$ & Sí & $\ldots$ & NA & Sí & No & No \\
\hline Costa Rica & Sí & Sí & Sí & $\ldots$ & NA & Sí & No & No \\
\hline Guatemala & Sí & Sí & Sí & No & NA & No & No & No \\
\hline Honduras & No & No & Sí & No & NA & Sí & No & Sí \\
\hline Suriname & Sí & Sí & Sí & No & NA & No & No & Sí \\
\hline Uruguaya $^{\mathrm{a}}$ & Sí & Sí & Sí & No & $\begin{array}{l}\text { Base imponible fija } \\
\text { "precios fictos" }\end{array}$ & $\mathrm{No}^{\mathrm{g}}$ & No & No \\
\hline \multicolumn{9}{|c|}{ Impuesto selectivo con estructura combinada } \\
\hline Dominica & No & Sí & Sí & No & Precio de fábrica & No & No & No \\
\hline Ecuador & No & Sí & Sí & No & $\begin{array}{l}\text { Precio minorista } \\
\text { excluido IVA e imp. } \\
\text { selectivo }\end{array}$ & Sí & Sí & No \\
\hline \multicolumn{9}{|c|}{ Impuesto selectivo con estructura mixta ${ }^{c}$} \\
\hline El Salvador & No & Sí & Sí & No & $\begin{array}{l}\text { Precio minorista } \\
\text { excluido IVA e imp. } \\
\text { selectivo }\end{array}$ & $\mathrm{No}^{\mathrm{h}}$ & No & No \\
\hline México & No & Sí & Sí & No & Precio de fábrica ${ }^{\dagger}$ & Sí & No & Sí \\
\hline
\end{tabular}

..: información no disponible

(1)

a Uruguay: El impuesto selectivo sobre las bebidas azucaradas está estructurado como impuesto ad valorem aplicado sobre una base imponible de montos fijos - "precios fictos" — por volumen, que varían por tipo de bebida, con lo cual funciona de hecho como un impuesto específico, y así se lo ha clasificado en el presente análisis.

Combinado: Por lo menos un tipo de bebida no alcohólica está gravado con un impuesto selectivo ad valorem y por lo menos otro tipo lo está con uno de monto específico. Ningún tipo de bebida está gravado con ambos.

' Mixto: Por lo menos un tipo de bebida no alcohólica está gravado tanto con un impuesto selectivo ad valorem como con uno de monto específico. En El Salvador y México únicamente las bebidas energizantes están gravadas por un sistema de impuesto selectivo mixto.

EInformación disponible únicamente para lurales están exentas de impuesto selectivo. casos, se pudo extraer la información de la legislación.

Mexico: El componente ad valorem se aplica únicamente a las bebidas energizantes.

"Uruguay: Los montos de la base imponible fija - "precios fictos"— se suelen ajustar anualmente, pero eso no es un requisito dispuesto por ley.

${ }^{\text {h }}$ El Salvador: El componente de monto específico se aplica únicamente a las bebidas energizantes.

¿ Chile y Perú: Diseño escalonado con distintas tasas tributarias ad valorem definidas por umbrales de concentración de azúcar.

Nicaragua: La tasa tributaria ad valorem es uniforme para las bebidas azucaradas, pero al agua mineral se le aplica una tasa menor.

Fuente: Preparado por los autores sobre la base de los datos del estudio. 
tributarias múltiples (diseño escalonado), en lugar de una sola tasa para todas las bebidas azucaradas sujetas al impuesto selectivo (diseño uniforme). Las tasas escalonadas se definen con mayor frecuencia por tipo de bebida o código arancelario armonizado. Otros ejemplos incluyen diseños escalonados definidos por umbrales de concentración de azúcar (por ejemplo, Perú y Chile), una combinación de tipo de bebida y contenido de azúcar (por ejemplo, Ecuador) o una combinación de tipo de bebida y concentración de jugo de fruta (por ejemplo, Argentina). Diez países aplican impuestos selectivos uniformes (cuadro 2).

\section{Base imponible}

Para los impuestos selectivos ad valorem, la base imponible se define como el valor del producto gravado, que se puede aplicar en distintos tramos de la cadena de valor. En los casos en que la base imponible para las bebidas de producción local se determina en fases iniciales de dicha cadena, como el precio de fábrica, según ocurre en cinco países (Barbados, Brasil, Dominica, México y Paraguay), las tasas del impuesto ad valorem se aplican a un valor menor, lo cual reduce el impacto del impuesto en los precios minoristas finales. Los otros nueve países que tienen un componente ad valorem usan una base imponible para las bebidas de producción local que se define en tramos posteriores de la cadena de valor, más cerca del precio minorista final (cuadro 2). En el caso de las bebidas importadas, los países que aplican impuestos selectivos ad valorem utilizan como base imponible el valor de costo, seguro y flete (CIF, por su sigla en inglés) y, cuando corresponde, los derechos de importación y aduaneros. El valor CIF se usa en la mayoría de los casos como base para los derechos de importación y se define como el valor de la consignación sin descargar, que comprende los costos del propio producto, del seguro y del transporte y descarga. Esa base imponible, establecida en tramos iniciales de la cadena de valor, reduce el impacto del impuesto en los precios minoristas finales.

En el caso de los impuestos selectivos de monto específico, la base imponible se puede definir según el volumen de la bebida o el contenido de azúcar. Casi todos los países con un componente de monto específico utilizan como base imponible el volumen de la bebida. Solamente Ecuador utiliza el contenido de azúcar como base imponible para su impuesto selectivo de monto específico de US\$ 0,18 por 100 gramos de azúcares para bebidas de más de 25 gramos de azúcares por litro (excepto las energizantes). Además, Chile y Perú utilizan un diseño escalonado para su impuesto selectivo ad valorem con distintas tasas tributarias definidas por umbrales de concentración de azúcar (cuadro 2).

\section{DISCUSIÓN}

La mayoría de los países de América Latina y el Caribe aplican impuestos selectivos sobre las bebidas azucaradas. Sin embargo, algunos de esos impuestos llevan más de un decenio sin actualizarse y muchos no están optimizados para alcanzar objetivos de salud.

\section{Consideraciones clave en el diseño de impuestos selectivos sobre las bebidas azucaradas}

Partiendo de la base de las mejores prácticas sobre la tributación del tabaco, establecimos algunas consideraciones clave para las distintas estructuras de impuestos selectivos observadas. Se ha demostrado que los impuestos selectivos ad valorem sobre los productos tabacaleros amplían la brecha entre el precio de los productos menos y más caros, lo cual induce al consumidor a optar por marcas más baratas y menoscaba los potenciales beneficios de salud del impuesto (18). La evidencia emergente sugiere que los impuestos selectivos ad valorem sobre las bebidas azucaradas pueden tener un efecto similar (16). Además, los impuestos selectivos ad valorem aplicados en función de una base imponible establecida en tramos iniciales de la cadena de valor, como el precio de fábrica para las bebidas de producción local y el valor CIF para las importadas, tienen un impacto menor en el precio minorista final que si se aplican en función de una base imponible establecida en tramos posteriores de dicha cadena, como el precio minorista. Por otra parte, los impuestos selectivos de monto específico se aplican sobre la misma base imponible para las bebidas de producción local e importadas. Además, también reducen los incentivos para pasar a consumir marcas menos caras. No obstante, el valor real de los impuestos selectivos de monto específico y su eficacia para reducir el consumo tienden a disminuir con el tiempo si no son ajustados regularmente para tener en cuenta la inflación y también, idealmente, el aumento del ingreso (18).

Según la OMS, los impuestos selectivos calculados sobre la base del contenido de azúcar pueden tener una repercusión mayor. De hecho, crean una diferenciación en la carga impositiva entre las opciones basadas en el contenido de azúcar dentro de una categoría de productos y pueden inducir al consumidor a optar por bebidas de menor contenido de azúcar y, al mismo tiempo, alentar a los productores a reformular sus bebidas (5).

Partiendo del mismo razonamiento, desde el punto de vista de la salud, al agua embotellada no se le deberían aplicar impuestos selectivos, pues ello les resta a dichos impuestos la posibilidad de generar una diferencia de precio entre las bebidas azucaradas y las no azucaradas, y no induce al consumidor a abandonar las azucaradas en favor de una opción más saludable. Además, demuestra que, en algunos países, los impuestos selectivos sobre las bebidas azucaradas se han establecido para aumentar los ingresos fiscales, sin considerarlos como instrumento de políticas de salud.

Por último, la lista de productos imponibles debería incorporar todos los tipos de bebidas azucaradas, incluidas las bebidas lácteas azucaradas, así como los polvos, concentrados o jarabes usados para hacer bebidas azucaradas añadiendo agua no gasificada o gasificada, para prevenir las sustituciones no deseadas de bebidas azucaradas gravadas por otras no gravadas. La simplicidad y la transparencia en la estructura tributaria y la definición de los productos imponibles reducen las oportunidades de elusión fiscal.

\section{Oportunidades para centrarse en la salud}

Habida cuenta de las consideraciones anteriores, los impuestos selectivos existentes se podrían modificar a fin de mejorar su eficacia para reducir el consumo de bebidas azucaradas, con medidas como recurrir más a los impuestos de monto específico, gravar en función del contenido de azúcar, aumentar las tasas tributarias, excluir el agua embotellada de la lista de productos imponibles y cerrar las lagunas que incentivan las sustituciones no deseables al incluir explícitamente en dicha lista todas las categorías de bebidas azucaradas: bebidas gaseosas azucaradas, 
bebidas con sabor a fruta, jugos de fruta, bebidas deportivas y energizantes, bebidas de agua vitaminada, tés helados y limonadas edulcoradas, bebidas lácteas y yogures azucarados, así como polvos, concentrados o jarabes usados para hacer bebidas azucaradas añadiendo agua sin o con gas.

En los países que no aplican impuestos selectivos sobre las bebidas azucaradas, la comunidad de salud pública tiene la oportunidad de abogar por su introducción y de asegurarse de que se diseñen de modo de optimizar los resultados de salud desde el principio.

\section{Necesidad de mejores prácticas en materia de tributación de las bebidas azucaradas}

Entre los actuales impuestos selectivos sobre las bebidas azucaradas hay una gran heterogeneidad y poca orientación en cuanto a las mejores prácticas en la tributación de dichas bebidas en comparación con la orientación para la tributación del tabaco y las bebidas alcohólicas $(18,24)$. Claramente, hay que seguir elaborando mejores prácticas basadas en datos empíricos para diseños eficaces de impuestos selectivos sobre las bebidas azucaradas. Las investigaciones futuras deberían estar dirigidas a evaluar la repercusión que diferentes diseños de impuestos selectivos tienen sobre los precios, el consumo, los ingresos tributarios y los resultados de salud, y la medida en que distintos diseños se podrían vincular con consecuencias no deseadas.

\section{Necesidad de un seguimiento sistemático de la tributación de las bebidas azucaradas}

Es necesario establecer sistemas de seguimiento periódico y estandarizado para captar los cambios en los niveles de tributación aplicados a las bebidas azucaradas en el transcurso del tiempo y poder efectuar comparaciones entre países. Para ello, partiendo de la experiencia de la OMS en cuanto al seguimiento de la tributación del tabaco, la OPS está trabajando en este momento a la elaboración de un indicador estandarizado sobre el porcentaje que los impuestos indirectos (incluidos los impuestos al valor agregado, selectivos y derechos aduaneros y de importación) representan en los precios minoristas de las bebidas azucaradas en América Latina y el Caribe (25).

\section{Limitaciones}

En nuestro análisis no proporcionamos información sobre las tasas de los impuestos selectivos establecidas en la legislación. De hecho, las comparaciones entre países pueden resultar engañosas a menos que se tengan en cuenta las diferencias en cuanto a la estructura del impuesto, la base imponible, el precio, el contenido de azúcar y el volumen de la bebida. Si bien no nos centramos en las tasas establecidas de los impuestos selectivos, cabe destacar que dichas tasas deben ser suficientemente elevadas para ser un desincentivo efectivo que induzca al consumidor a no comprar bebidas azucaradas $(5,20,26)$.

Aun cuando analizamos si los impuestos selectivos existentes se aplican especialmente a ciertas categorías de bebidas no alcohólicas, no hemos evaluado la tributación de las bebidas con sabor a fruta, los jugos de frutas o las bebidas deportivas. $\mathrm{Ni}$ dichos jugos ni las bebidas deportivas formaron parte de las bebidas para las cuales la encuesta de la OPS sobre los impuestos a las bebidas azucaradas recopiló información tributaria.
Por otra parte, si bien dicha encuesta recogió información sobre la tributación de las bebidas con sabor a fruta, la información fue difícil de verificar en la legislación debido a la definición amplia del código arancelario armonizado, partida 2009, que incluye los jugos de fruta "con o sin adición de azúcar u otro edulcorante" (23).

Por último, los datos presentados en nuestro análisis están basados en legislación que estaba en vigor al 31 de marzo del 2019. La legislación que pudiera haber sido reemplazada, enmendada o derogada desde esa fecha de corte no fue analizada, de manera de mantener la comparabilidad de los datos en el mismo momento en todos los países. Desde esa fecha de corte no detectamos la introducción de ningún nuevo impuesto selectivo sobre las bebidas azucaradas en América Latina y el Caribe.

\section{Conclusión}

Al mes de marzo del 2019, 21 países de América Latina y el Caribe aplicaban impuestos selectivos sobre las bebidas azucaradas. Si bien ello es prometedor, la mayoría de esos impuestos se podrían aprovechar aún más a fin de que tengan un mayor impacto sobre el consumo de bebidas azucaradas y la salud. Esto ofrece la oportunidad de proponer enmiendas a los impuestos selectivos existentes y abogar por su introducción en los países que actualmente no los aplican.

El diseño de los impuestos selectivos existentes sobre las bebidas azucaradas es sumamente diverso. Todos los países se beneficiarían de orientación adicional en cuanto a la manera de introducir o enmendar dichos impuestos para optimizar los beneficios de salud. Vista la carga mundial de las ENT tanto actual como proyectada, será importante dar estrecho seguimiento al uso de esa política eficaz a nivel poblacional y aumentar al máximo su potencial de salud.

Contribuciones de los autores. RCS conceptualizó el estudio. Todos los autores recopilaron los datos. MR, IB, MA, LG y GP analizaron los datos e interpretaron los resultados con aportes de todos los autores. MR, IB y MA redactaron el manuscrito y todos los autores los revisaron de manera crítica. Todos ellos también revisaron y aprobaron la versión definitiva.

Agradecimientos. Deseamos agradecer y reconocer las valiosas aportaciones del Departamento de Enfermedades No Transmisibles y Salud Mental de la OPS, especialmente Anselm Hennis, Adriana Blanco, Rubén Grajeda, Maristela Monteiro, Elisa Prieto, Alicia Nicholls, Patty Welch, Alejandro Álvarez y Steven Constantinou, así como del O'Neill Institute for National and Global Health Law de la Universidad de Georgetown. Un agradecimiento especial a todos los funcionarios de los ministerios de economía de América Latina y el Caribe que participaron en la encuesta. También agradecemos y reconocemos el apoyo de Global Health Advocacy Incubator y el financiamiento aportado por Bloomberg Philanthropies.

\section{Conflictos de intereses. Ninguno declarado.}

Declaración. Los autores son los únicos responsables de las opiniones expresadas en el manuscrito, que pueden no reflejar necesariamente la opinión o la política de la RPSP/PAJPH o de la OPS. 


\section{REFERENCIAS}

1. Organización Panamericana de la Salud. (2019). Enfermedades no transmisibles en la Región de las Américas: hechos y cifras [Internet]. Washington, D.C.: OPS; 2019 [consultado el 23 de marzo del 2020]. Disponible en: https://iris.paho.org/handle/10665.2/51482.

2. Bloom D, Cafiero E, Jané-Llopis E, Abrahams-Gessel S, Bloom L, Fathima S, et al. The Global Economic Burden of Non-communicable Diseases [Internet]. Ginebra: Foro Económico Mundial; 2011 [consultado el 18 de abril del 2019]. Disponible en: http://www3. weforum.org/docs/WEF_Harvard_HE_GlobalEconomicBurden NonCommunicableDiseases_2011.pdf

3. Organización Mundial de la Salud. Lucha contra las ENT: "mejores inversiones" y otras intervenciones recomendadas para la prevención y el control de las enfermedades no transmisibles [Internet]. Ginebra: OMS; 2017 [consultado el 18 de abril del 2019]. Disponible en: https:/ /apps.who.int/iris/handle/10665/259351.

4. Organización Mundial de la Salud. Technical Annex: Updated Appendix 3 of the WHO Global NCD Action Plan 2013-2020 [Internet]. Ginebra: OMS; 2017 [consultado el 4 de marzo del 2019]. Disponible en: https://www.who.int/ncds/governance/technical_annex.pdf.

5. Organización Mundial de la Salud. Fiscal policies for diet and prevention of noncommunicable diseases: technical meeting report, 5 y 6 de mayo del 2015, Ginebra (Suiza) [Internet]. Ginebra: OMS; 2016 [consultado el 20 de abril del 2019]. Disponible en: https://apps. who.int/iris/handle/10665/250131.

6. Woodward-Lopez G, Kao J, Ritchie L. To what extent have sweetened beverages contributed to the obesity epidemic? Public Health Nutrition. Marzo del 2011;14(3):499-509.

7. Brownell KD, Frieden TR. Ounces of prevention-the public policy case for taxes on sugared beverages. N Engl J Med. 30 de abril del 2009;360(18):1805-8.

8. Malik VS, Pan A, Willett WC, Hu FB. Sugar-sweetened beverages and weight gain in children and adults: a systematic review and meta-analysis. Am J Clin Nutr. 1 de octubre del 2013;98(4):1084-102.

9. Xi B, Huang Y, Reilly KH, Li S, Zheng R, Barrio-Lopez MT, Martínez-González MA, Zhou D. Sugar-sweetened beverages and risk of hypertension and CVD: a dose-response meta-analysis. Br J Nutr. Marzo del 2015;113(5):709-17.

10. Imamura F, O'Connor L, Ye Z, Mursu J, Hayashino Y, Bhupathiraju SN, et al. Consumption of sugar sweetened beverages, artificially sweetened beverages, and fruit juice and incidence of type 2 diabetes: systematic review, meta-analysis, and estimation of population attributable fraction. Br J Sports Med. 1 de abril del 2016;50(8):496-504.

11. Singh GM, Micha R, Khatibzadeh S, Lim S, Ezzati M, Mozaffarian D. Estimated global, regional, and national disease burdens related to sugar-sweetened beverage consumption in 2010. Circulation. 25 de agosto del 2015;132(8):639-66.

12. Singh GM, Micha R, Khatibzadeh S, Shi P, Lim S, Andrews KG, et al. Global, regional, and national consumption of sugar-sweetened beverages, fruit juices, and milk: a systematic assessment of beverage intake in 187 countries. PLoS One. 5 de Agosto del 2015;10(8):e0124845.

13. Legetic B, Medici A, Hernández-Avila M, Alleyne G, Hennis A, editores. Economic Dimensions of Noncommunicable Diseases in Latin America and the Caribbean. Disease Control Priorities, 3rd ed. [Internet]. Washington, DC: Organización Panamericana de la Salud; 2016 [consultado el 17 de octubre del 2019]. Disponible en: https://iris.paho.org/handle/10665.2/28501.

14. Colchero MA, Rivera-Dommarco J, Popkin BM, Ng SW. In Mexico, evidence of sustained consumer response two years after implementing a sugar-sweetened beverage tax. Health Aff (Millwood). 1 de marzo del 2017;36(3):564-71.

15. CaroJC, Corvalán C, Reyes M, Silva A, Popkin B, Taillie LS. Chile's 2014 sugar-sweetened beverage tax and changes in prices and purchases of sugar-sweetened beverages: An observational study in an urban environment. PLoS Medicine. 3 de julio del 2018;15(7):e1002597.

16. Alvarado M, Unwin N, Sharp SJ, Hambleton I, Murphy MM, Samuels TA, et al. Assessing the impact of the Barbados sugar-sweetened beverage tax on beverage sales: an observational study. Int J Behav Nutr Phys Act. 30 de enero del 2019;16(1):13.

17. Barrientos-Gutierrez T, Zepeda-Tello R, Rodrigues ER, Colchero-Aragonés A, Rojas-Martínez R, Lazcano-Ponce E, et al. Expected population weight and diabetes impact of the 1-peso-per-litre tax to sugar sweetened beverages in Mexico. PLoS One. 17 de mayo del 2017;12(5):e0176336.

18. Organización Panamericana de la Salud. Manual técnico de la OMS sobre Administración de impuestos al tabaco [Internet]. Washington, DC: OPS; 2015 [consultado el 15 de febrero del 2019]. Disponible en: https://www.paho.org/es/documentos/manualtecnico-oms-sobre-administracion-impuestos-al-tabaco.

19. Organización Mundial de la Salud. Report on the global tobacco epidemic, 2017: monitoring tobacco use and prevention policies. Technical Note III: Tobacco taxes in WHO Member States [Internet] Ginebra: OMS; 2017 [consultado el 15 de febrero del 2019]. Disponible en: https://apps.who.int/iris/handle/10665/255874.

20. Organización Panamericana de la Salud/Organización Mundial de la Salud. Impuestos saludables: guía breve (documento de la OMS sobre políticas) [Internet]. Washington, DC: OPS; 2020 [consultado el 23 de marzo del 2020]. Disponible en: https://iris.paho.org/ handle/10665.2/52647.

21. Thow AM, Downs SM, Mayes C, Trevena H, Waqanivalu T, Cawley J. Fiscal policy to improve diets and prevent noncommunicable diseases: from recommendations to action. Bull World Health Org. 1 de marzo del 2018;96(3):201-10.

22. Organización Mundial del Comercio. Trade Policy Review. Report by the Secretariat: Haiti. Revision. Trade Policy Review Body. WT/ TPR/S/327/ Rev.1 [Internet]. Ginebra: OMC; 2016 [consultado el 15 de abril del 2020]. Disponible en: https://docs.wto.org/dol2fe/ Pages /SS/directdoc.aspx?filename=q:/WT/TPR/S327R1.pdf.

23. Organización Mundial de Aduanas. HS Nomenclature 2017 edition [Internet]. Bruselas: OMA; 2017 [consultado el 21 de mayo del 2020]. Disponible en: http://www.wcoomd.org/en/topics/ nomenclature/instrument-and-tools/hs-nomenclature-2017edition/hs-nomenclature-2017-edition.aspx.

24. Sornpaisarn B, Shield KD, Österberg E, Rehm J. Herramienta sobre políticas tributarias y de precios del alcohol [Internet]. Washington, DC: Organización Panamericana de la Salud; 2019 [consultado el 17 de octubre del 2019]. Disponible en: https://www.paho.org/es/ documentos/herramienta-sobre-politicas-tributarias-precios-alcohol.

25. Organización Panamericana de la Salud. Reunión para desarrollar un indicador estandarizado del porcentaje que los impuestos representan en los precios de las bebidas alcohólicas y bebidas azucaradas (Washington, D.C., 24 y 25 de julio del 2018) [Internet]. Washington, DC: OPS; 2021 [consultado el 6 de agosto del 2021]. Disponible en: https://iris.paho.org/handle/10665.2/54404.

26. Powell LM, Chriqui JF, Khan T, Wada R, Chaloupka FJ. Assessing the potential effectiveness of food and beverage taxes and subsidies for improving public health: a systematic review of prices, demand and body weight outcomes: Review of prices, demand and weight. Obes Rev. Febrero del 2013;14(2):110-28.

Manuscrito (original en inglés) recibido el 12 de octubre del 2020. Versión revisada aceptada para su publicación el 1 de diciembre del 2020. 


\section{Excise taxes on sugar-sweetened beverages in Latin America and the Caribbean}

Abstract

Keywords
Objective. To characterize the design of excise taxes on sugar-sweetened beverages (SSBs) in Latin America and the Caribbean and assess opportunities to increase their impact on SSB consumption and health.

Methods. A comprehensive search and review of the legislation in effect as of March 2019, collected through existing Pan American Health Organization and World Health Organization monitoring tools, secondary sources, and surveying ministries of finance. The analysis focused on the type of products taxed, and the structure and base of these excise taxes.

Results. Out of the 33 countries analyzed, 21 apply excise taxes on SSBs. Seven countries also apply excise taxes on bottled water and at least four include sugar-sweetened milk drinks. Ten of these excise taxes are ad valorem with some tax bases set early in the value chain, seven are amount-specific, and four have either a combined or mixed structure. Three countries apply excise taxes based on sugar concentration.

Conclusions. While the number of countries applying excise taxes on SSBs is promising, there is great heterogeneity in design in terms of structure, tax base, and products taxed. Existing excise taxes could be further leveraged to improve their impact on SSB consumption and health by including all categories of SSBs, excluding bottled water, and relying more on amount-specific taxes regularly adjusted for inflation and possibly based on sugar concentration. All countries would benefit from additional guidance. Future research should aim to address this gap. Keywords Noncommunicable diseases; nutrition policy; health economics; obesity; legislation as topic

Noncommunicable diseases; nutrition policy; health economics; obesity; legislation as topic

\section{Imposto especial de consumo sobre bebidas açucaradas na América Latina e no Caribe}

RESUMO Objetivo. Caracterizar o modelo dos impostos especiais de consumo sobre bebidas açucaradas na América Latina e no Caribe e avaliar oportunidades para aumentar o impacto desses impostos no consumo de bebidas açucaradas e na saúde.

Métodos. Realizou-se uma pesquisa ampla e a análise de legislações vigentes em março de 2019, com informações obtidas por meio de instrumentos de monitoramento da Organização Pan-Americana da Saúde (OPAS) e da Organização Mundial da Saúde (OMS) já existentes, fontes secundárias e levantamento junto aos ministérios da Fazenda. A análise centrou-se no tipo de produtos tributados e na estrutura e base desses impostos especiais de consumo.

Resultados. Dos 33 países analisados, 21 aplicam impostos especiais de consumo sobre bebidas açucaradas. Em sete países os impostos especiais de consumo incidem também sobre água engarrafada e, em pelo menos quatro, incluem bebidas lácteas açucaradas. Dez desses tributos especiais são ad valorem com algumas bases tributárias estabelecidas no início da cadeia de valor, sete são de tipo específico e quatro têm uma estrutura combinada ou mista. Em três países os impostos especiais são estabelecidos com base na concentração de açúcares do produto.

Conclusões. Apesar do número promissor de países com impostos especiais de consumo sobre bebidas açucaradas, verifica-se grande heterogeneidade nos modelos de tributação em termos de estrutura, base tributária e produtos tributados. Os impostos especiais de consumo vigentes poderiam ser mais bem aproveitados para aumentar o impacto no consumo de bebidas açucaradas e na saúde: incluir todas as categorias de bebidas açucaradas, excluir água engarrafada e recorrer mais a impostos de tipo específico com a correção periódica pela inflação e, possivelmente, com base na concentração de açúcares do produto. Todos os países se beneficiariam em receber mais orientação. Pesquisas futuras devem ter como objetivo abordar essa lacuna.

Palavras-chave Doenças não transmissíveis; política nutricional; economia da saúde; obesidade; legislação como assunto. 